\title{
Coronavirus disease (COVID-19) with orbital complication in young child
}

\author{
Florian Schmidt ${ }^{1}$ and Harish Viswanathan ${ }^{1}$ \\ ${ }^{1}$ Portsmouth Hospitals NHS Trust
}

April 28, 2020

\section{Coronavirus disease (COVID-19) with orbital complication in young child}

- COVID-19 is a current ongoing global health emergency

- Coronaviruses cause sinonasal symptoms similar to common cold and loss of smell

- Endoscopic sinus surgery is an aerosol-generating procedure with high risk of COVID-19 transmission

- COVID-19 infection was related to a case of preseptal orbital cellulitis

- Covid-19 infection should be suspected in current cases of orbital cellulitis and management plan modified to protect medical professionals

\section{Introduction}

The novel coronavirus disease 2019 (COVID-19) is a current cataclysmic ongoing global health emergency with rapidly increasing case numbers. Coronaviruses cause symptoms ranging from those similar to the common cold to severe respiratory symptoms and COVID-19 is reported to be highly infectious. To our knowledge this is the first case report of an orbital complication related to acute rhinosinusitis secondary to new coronavirus disease.

\section{Case presentation}

A three year and five month old boy presented recently to our ENT emergency service with a two day history of left sided eye swelling and redness. He had a raised temperature of 39 degree on admission day. Parents reported him to have corysal symptoms of upper respiratory tract infection with nasal congestion, green rhinorrhoea and cough for the past ten days. His past medical history was not significant apart from pre-mature birth at week 32 by C-section due to pre-eclampsia and subsequent neonatal intensive care stay due to intrauterine growth restriction but normal development since.

On examination left upper and lower lid showed severe inflammatory swelling with inability to open the eye voluntarily. There was no chemosis and he had normal eye movement without proptosis. Accurate assessment of visual acuity was not tolerated.

Biochemically he had raised white blood cell count of $25.910 * 9 / \mathrm{L}$, Neutrophils of 20.7, Lymphocytes 2.1 and C-reactive protein of $200 \mathrm{mg} / \mathrm{L}$. COVID-19 Polymerase chain reaction testing came back positive on day 2 of admission and additional bacterial culture from nasal secretion showed Group A haemolytic streptococci.

Based on initial physical examination a diagnosis of preseptal cellulitis consistent with Grade I Chandler classification (Figure 1) was established. We started intravenous antibiotics with Ceftriaxone and Metronidazole alongside Xylometazoline nasal drops and Paracetamol.

Clinical re-assessment after 24 and 48 hours revealed significant clinical improvement of the orbital swelling (Figure 2). The child remained afebrile and improved biochemically. No imaging or surgical intervention 
was required. He was discharged to complete a course of oral antibiotics with normal eye examination on day 5 .

\section{Discussion}

The novel coronavirus disease 2019 caused by severe acute respiratory syndrome coronavirus 2 is a current cataclysmic ongoing global health emergency with rapidly increasing case numbers. Current epidemiology concentrates on adult patients with fewer cases reported in children ${ }^{1}$. Coronaviruses are enveloped singlestranded Ribonucleic acid viruses that cause symptoms ranging from those similar to the common cold to severe respiratory symptoms ${ }^{2}$. Recently ENTUK stated evidence for the loss of sense of smell as a symptom of COVID-19 infection in more than two out of three patients suggesting relevant sinonasal involvement of the disease ${ }^{3}$.

To our knowledge this is the first case report of an orbital complication related to coronavirus disease. We think bacterial superinfection with Group A haemolytic streptococci on top of a coronavirus infection led to orbital cellulitis in this case.

More advanced orbital infections require surgical intervention to drain collections and prevent loss of visual acuity, neurological complications or even lethal outcomes. Conventionally urgent endoscopic and/or external sinonasal surgical approaches are required from Chandler stage III onwards. However endoscopic procedures have now anecdotally been reported to be highly infectious for surgeons and theatre personnel due to high viral load in the nasal cavity leading to increased viral shedding and possible aerosoliziation of viral particles during these procedures4. This might place ear, nose and throat surgeons and theatre personal at significant risk of infection. BAPO (British Association of Paediatric Otolaryngology) suggests limiting surgical intervention to cases where vision is at risk and conservative measures have failed5. The recommendation is to perform surgery via external approach in these cases.

\section{Conclusion}

To our knowledge, this is the first case report of an orbital cellulitis related to new coronavirus disease in a young child. Ear, nose and throat surgeons are commonly dealing with orbital complications in their emergency services and often rapid sinus surgery is required to prevent serious harm. Covid-19 infection should be suspected in all current case of orbital cellulitis and management plan modified to protect the medical personnel from potentially fatal infection.

\section{References}

1. Adhikari SP, Meng S, Wu YJ, Mao YP, Ye RX, Wang QZ, Sun C, Sylvia S, Rozelle S, Raat H, Zhou H. Epidemiology, causes, clinical manifestation and diagnosis, prevention and control of coronavirus disease (COVID-19) during the early outbreak period: a scoping review. Infect Dis Poverty. 2020 Mar $17 ; 9(1): 29$.

2. Zhu N, Zhang D, Wang W, Li X, Yang B, Song J, Zhao X, Huang B, Shi W, Lu R, Niu P, Zhan F, Ma X, Wang D, Xu W, Wu G, Gao GF, Tan W; China Novel Coronavirus Investigating and Research Team. A Novel Coronavirus from Patients with Pneumonia in China, 2019. N Engl J Med. 2020 Feb $20 ; 382(8): 727-733$.

3. ENTUK. COVID-19 Information for health professionals. https://www.entuk.org/sites/default/files/files/Loss\%20of\%2

4. Stanford University School of Medicine Departments of Otolaryngology-Head \& Neck Surgery and Neurosurgery. Precautions for endoscopic transnasal skull base surgery during the COVID-19 pandemic. 12 March 2020. https://www.enttoday.org/article/otolaryngologists-may-contract-covid-19during-surgery/

5. ENTUK. COVID-19 Information for health professionals. https://www.entuk.org/sites/default/files/files/BAPOstatem

\section{Figures}

1. Figure 1. Chandler classification of orbital complications of sinusitis from https://www.enteducationswansea.org/rhinosi

2. Figure 2. Photograph patient with left preseptal orbital cellulitis 


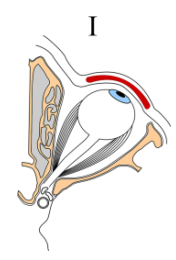

Preseptal cellulitis

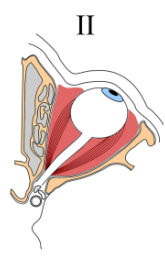

Orbital cellulitis

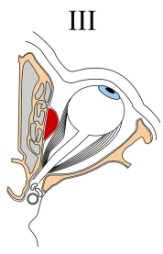

Subperiosteal Abscess

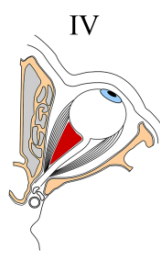

Orbital Abscess

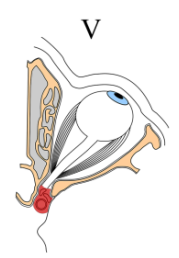

Cavernous Sinus Thrombosis

Chandler's Classification

Figure 1. Chandler classification of orbital complications of sinusitis (with kind approval from https://www.enteducationswar

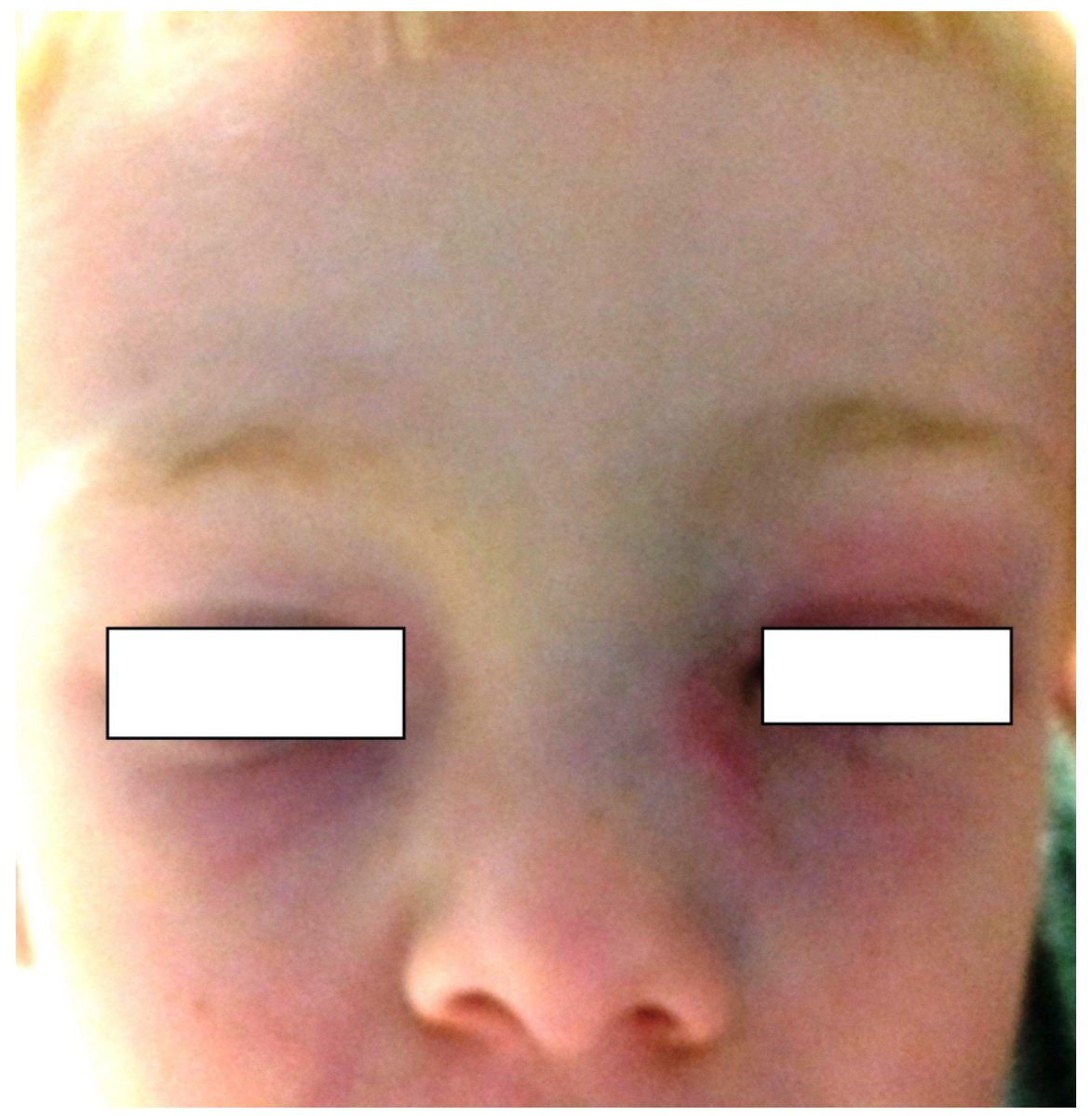

Figure 2. Patient with left preseptal orbital cellulitis after 48 hours of intravenous antibiotics. 